\title{
Heterogeneity of Intrinsic and Synaptic Properties of Neurons in the Ventral and Dorsal Parts of the Ventral Nucleus of the Lateral Lemniscus
}

\author{
Franziska Caspari ${ }^{\dagger}$, Veronika J. Baumann ${ }^{\dagger}$, Elisabet Garcia-Pino and Ursula Koch* \\ Neurophysiology, Institute of Biology, Freie Universität Berlin, Berlin, Germany
}

OPEN ACCESS

Edited by:

Manuel S. Malmierca, University of Salamanca, Spain

Reviewed by:

Diasynou Fioravante, University of California, Davis, USA Huiming Zhang,

University of Windsor, Canada

*Correspondence: Ursula Koch ursula.koch@fu-berlin.de

tThese authors have contributed equally to this work.

Received: 22 July 2015 Accepted: 30 October 2015

Published: 18 November 2015

Citation:

Caspari F, Baumann VJ, Garcia-Pino E and Koch U (2015) Heterogeneity of Intrinsic and Synaptic Properties of Neurons in the Ventral and Dorsal Parts of the Ventral Nucleus of the Lateral Lemniscus.

Front. Neural Circuits 9:74. doi: 10.3389/fncir.2015.00074
The ventral nucleus of the lateral lemniscus (VNLL) provides a major inhibitory projection to the inferior colliculus $(I C)$. Neurons in the VNLL respond with various firing patterns and different temporal precision to acoustic stimulation. The present study investigates the underlying intrinsic and synaptic properties of various cell types in different regions of the VNLL, using in vitro electrophysiological recordings from acute brain slices of mice and immunohistochemistry. We show that the biophysical membrane properties and excitatory input characteristics differed between dorsal and ventral VNLL neurons. Neurons in the ventral VNLL displayed an onset-type firing pattern and little hyperpolarization-activated current $\left(l_{h}\right)$. Stimulation of lemniscal inputs evoked a large all-or-none excitatory response similar to Calyx of Held synapses in neurons in the lateral part of the ventral VNLL. Neurons that were located within the fiber tract of the lateral lemniscus, received several and weak excitatory input fibers. In the dorsal VNLL onsettype and sustained firing neurons were intermingled. These neurons showed large $I_{h}$ and were strongly immunopositive for the hyperpolarization-activated cyclic nucleotidegated channel 1 (HCN1) subunit. Both neuron types received several excitatory inputs that were weaker and slower compared to ventrolateral VNLL neurons. Using a mouse model that expresses channelrhodopsin under the promotor of the vesicular GABA transporter (VGAT) suggests that dorsal and ventral neurons were inhibitory since they were all depolarized by light stimulation. The diverse membrane and input properties in dorsal and ventral VNLL neurons suggest differential roles of these neurons for sound processing.

Keywords: hearing, auditory brainstem, membrane properties, synaptic transmission, hyperpolarization-activated current

\section{INTRODUCTION}

The ventral nucleus of the lateral lemniscus (VNLL), an auditory brainstem nucleus of the ascending auditory pathways, might play a critical role in the analysis of temporal sound patterns (Covey and Casseday, 1991; Recio-Spinoso and Joris, 2014). Neurons in the VNLL receive glutamatergic, excitatory inputs from several neuron types of the contralateral ventral cochlear nucleus (VCN) 
and a major glycinergic, inhibitory input from the ipsilateral medial nucleus of the trapezoid body (MNTB; Glendenning et al., 1981; Schofield and Cant, 1997; Irfan et al., 2005; Kelly et al., 2009). Most VNLL neurons are immunopositive for glycine and/or GABA and send inhibitory projections to the ipsilateral inferior colliculus (IC; Saint Marie et al., 1997; Riquelme et al., 2001; Zhang and Kelly, 2006). Based on the large number of GABAergic and glycinergic neurons in the VNLL, this nucleus represents a prominent inhibitory source to the IC (Saint Marie and Baker, 1990).

Neurons in the VNLL show diverse responses to sounds regarding temporal response patterns and binaurality (Batra and Fitzpatrick, 1997; Nayagam et al., 2006; Zhang and Kelly, 2006; Recio-Spinoso and Joris, 2014). Studies on brain slices have revealed various firing patterns in response to depolarizing current injections (Wu, 1999; Zhao and Wu, 2001; Irfan et al., 2005) that resemble firing pattern of VNLL neurons in vivo (Zhang and Kelly, 2006). A subset of VNLL neurons specifically responds to the onset of a sound with very short latencies and extremely low jitter (Covey and Casseday, 1991; Batra and Fitzpatrick, 1999; Zhang and Kelly, 2006). Anatomical studies suggest that these VNLL neurons receive a major excitatory input that arises from the octopus cells of the contralateral VCN (Adams, 1997; Schofield and Cant, 1997). These projections contact ventral VNLL neurons with calyx-like synapses that mediate information with high temporal fidelity (Berger et al., 2014).

So far the intrinsic and synaptic properties of VNLL neurons and their spatial distribution within the VNLL have not been systematically studied in animals when neuronal properties are considered to be mature (Khurana et al., 2012). To identify whether different neuron types are systematically distributed within the VNLL, as shown for echo-locating bats (Vater and Feng, 1990; Huffman and Covey, 1995), we characterized the intrinsic and synaptic properties of VNLL neurons in acute brain slices of young adult mice, relative to their location within the VNLL. Here, we show that the VNLL is a heterogeneous nucleus with neurons in the ventral and dorsal parts of the VNLL differing from each other in terms of their membrane properties, hyperpolarization-activated cyclic nucleotide gated (HCN) current density and their synaptic input characteristics. In the ventrolateral part of the VNLL a subpopulation of neurons with fairly uniform membrane and synaptic properties exists. These neurons receive one large excitatory synaptic input resembling Calyx of Held synaptic properties in the MNTB.

\section{MATERIALS AND METHODS}

\section{Animals}

All experiments followed EU ethical guidelines and were carried out in accordance with protocols approved by the German federal authorities (Landesamt für Gesundheit und Soziales, State of Berlin).

All patch-clamp recordings and immunohistochemical labeling were performed in brain slices containing the VNLL of $\mathrm{C} 57 / \mathrm{Bl} 6 \mathrm{~J}$ mice at the age of postnatal day 22/23 (denoted as P22) except for the experiments illustrated in Figure 7. For these experiments VGAT-ChR2-YFP ${ }^{+}$[B6.Cg-Tg(Slc32a1COP4*H134R/EYFP)8Gfng/J; The Jackson Laboratory] mice at P22 were used to determine inhibitory regions within the lateral lemniscus as channelrhodopsin-associated YFP is only expressed in vesicular GABA transporter (VGAT)-expressing neurons, hence GABAergic or glycinergic neurons (Zhao et al., 2011).

\section{Slice Preparation}

The animals were decapitated under isoflurane anesthesia. The brains were removed and sliced in ice-cold oxygenated (95\% $\mathrm{O}_{2} / 5 \% \mathrm{CO}_{2}$ ) sucrose replacement solution containing (in $\mathrm{mM}$ ): $2.5 \mathrm{KCl}, 26 \mathrm{NaHCO}_{3}, 1.25 \mathrm{NaH}_{2} \mathrm{PO}_{4}, 6 \mathrm{MgCl}_{2}, 0.5 \mathrm{CaCl}_{2}$, 25 glucose, 200 sucrose ( $\mathrm{pH}$ 7.4). Transverse brainstem slices $(180 \mu \mathrm{m})$ including the VNLL were cut with a vibratome (VT1200S; Leica, Germany), collected and stored at $32^{\circ} \mathrm{C}$ for $15 \mathrm{~min}$ for recovery in oxygenated artificial cerebrospinal fluid (ACSF) containing (in $\mathrm{mM}$ ): $125 \mathrm{NaCl}, 2.5 \mathrm{KCl}, 1.25$ $\mathrm{NaH}_{2} \mathrm{PO}_{4}, 26 \mathrm{NaHCO}_{3}, 1 \mathrm{MgCl}_{2}, 2 \mathrm{CaCl}_{2}, 25$ glucose and then kept at room temperature until recording for up to 4-5 h. For patch clamp experiments, slices were transferred to a recording chamber, which was perfused continuously with oxygenated ACSF at near-physiological temperature of $32^{\circ} \mathrm{C}$. An upright microscope (Axioscope, Zeiss, Germany) with infrareddifferential interference contrast optics was attached to visualize the neurons.

\section{Electrophysiology}

Whole-cell patch-clamp recordings were made from visually identified VNLL neurons using a Multiclamp 700 A amplifier (Axon Instruments, USA). Patch pipettes were pulled from borosilicate glass capillaries (BioMedical Instruments, Germany, $0.86 \mathrm{~mm}$ inner diameter and $1.5 \mathrm{~mm}$ outer diameter) on a DMZ Universal Puller (Zeitz Instruments, Germany) and filled with the following solutions: for current clamp recordings and hyperpolarization-activated current $\left(\mathrm{I}_{\mathrm{h}}\right)$ measurements: (in $\mathrm{mM}$ ) 125 K-gluconate, $5 \mathrm{KCl}, 10 \mathrm{HEPES}, 1$ EGTA, $2 \mathrm{Na}_{2} \mathrm{ATP}, 2$ MgATP, $0.3 \mathrm{Na}_{2}$ GTP, $10 \mathrm{Na}$-phosphocreatinine ( $\mathrm{pH}$ 7.25); for recordings of synaptic currents: $41 \mathrm{CsCl}, 99 \mathrm{Cs}$-methylsulfonate, 10 HEPES, 10 Cs-EGTA, $2 \mathrm{Na}_{2}$ ATP, 2 MgATP, $0.3 \mathrm{Na}_{2} \mathrm{GTP}$, $1 \mathrm{CaCl}_{2}, 5$ TEA-Cl, 5 QX 314 (pH 7.4). All experiments were performed at $32^{\circ} \mathrm{C}$. Patch pipettes had resistances between 2-4 M $\Omega$. To study the intrinsic membrane properties with respect to their location within the VNLL, the VNLL was subdivided into three regions: dorsal, middle and ventral based on the nomenclature proposed by Benson and Cant (2008) for gerbils. We mainly recorded from the ventral and dorsal parts of the VNLL (vVNLL and dVNLL, respectively; Figure 1A). The ventral VNLL was considered as the ventral end of the fiber bundle of the lateral lemniscus and the dorsal VNLL was determined with respect to the dorsal nucleus of the lateral lemniscus (DNLL). The neurons location was determined in the acute slice preparation by anatomical landmarks and delineated in a sketch of the VNLL. In some experiments Alexa 555 was added to the internal solution in order to later 


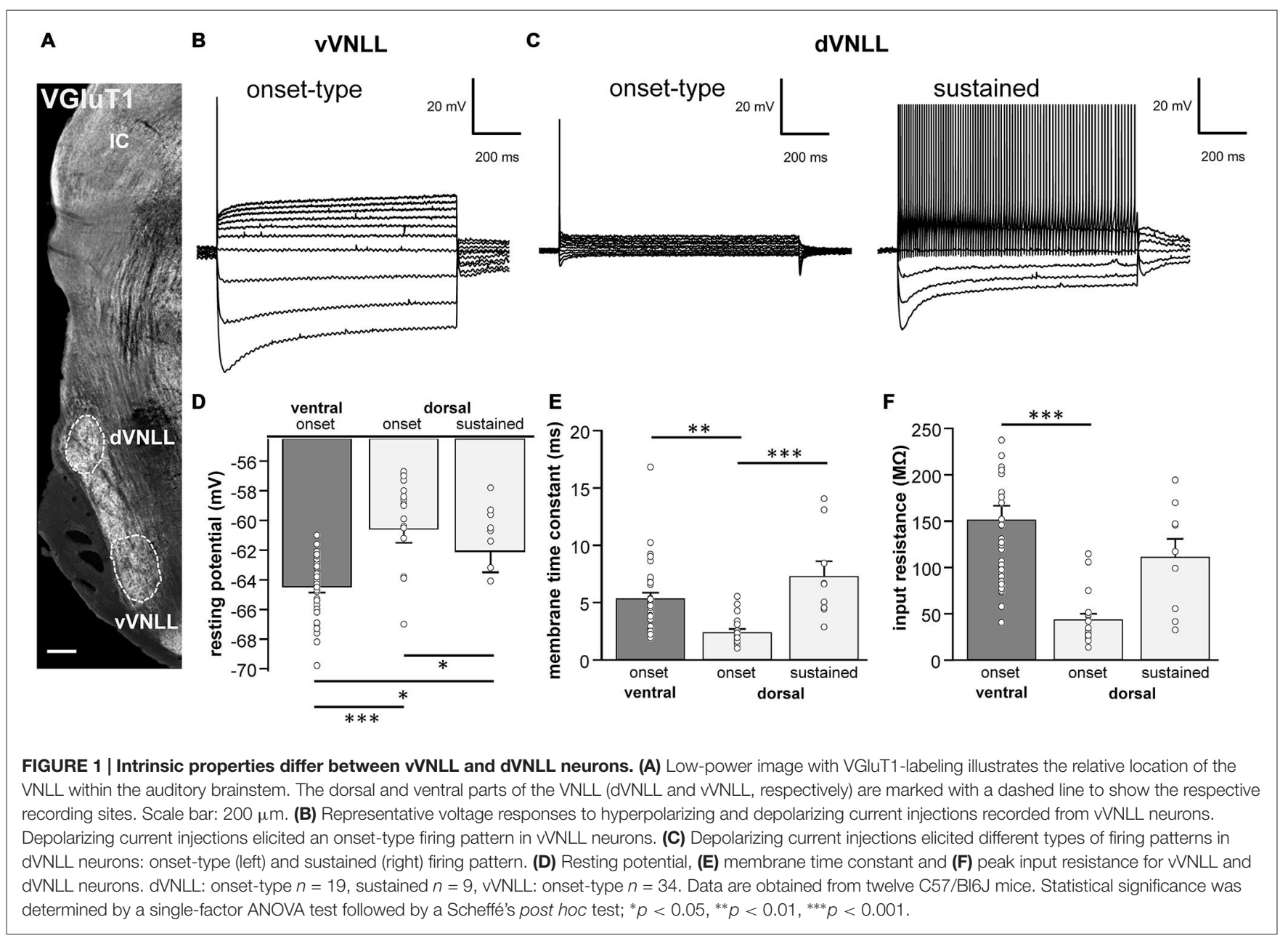

verify the visually determined location of a neuron within the VNLL.

Bridge balance was adjusted for current-clamp recordings. During voltage-clamp recordings series resistance $(<10 \mathrm{M} \Omega$ ) was compensated to a residual resistance of $2-2.5 \mathrm{M} \Omega$ and was not allowed to change more than $20 \%$ during the recording. For measuring passive and active membrane properties $1000 \mathrm{~ms}$ long hyperpolarizing and depolarizing current steps from -300 pA to $1000 \mathrm{pA}$ were injected. Channelrhodopsin was activated with a 5-10 ms light pulse of the Arc lamp which yields similar depolarization amplitude of VGAT-positive neurons as a 26.3 $\mathrm{mW} / \mathrm{mm}^{2}$ blue LED (Zhao et al., 2011).

According to Baumann et al. (2013) HCN-currents were isolated by pharmacological blockade of other ionic currents by applying the following drugs to the bath (in $\mathrm{mM}$ ): 1 3, 4-diaminopyridine, 10 TEA-Cl, $0.2 \mathrm{BaCl}_{2}, 0.001$ TTX, $0.05 \mathrm{NiCl}_{2}, 0.1 \mathrm{CdCl}_{2}, 0.01 \mathrm{DNQX}, 0.025$ DL-AP5 and 0.001 strychnine. The $\mathrm{NaCl}$ concentration was reduced to maintain iso-osmolarity. $\mathrm{I}_{\mathrm{h}}$ was activated by applying depolarizing and hyperpolarizing voltage steps from -40.5 to $-120.5 \mathrm{mV}$. Tail currents were measured at a holding potential of $-100.5 \mathrm{mV}$ that followed each voltage step. Current densities were obtained by estimating cell surface size from the capacitance compensation measurements of the amplifier.

Synaptic currents were evoked by stimulating the ascending fibers $50 \mu \mathrm{m}$ ventral to the recorded neuron with a glass electrode filled with $2 \mathrm{M} \mathrm{NaCl}$. Stimuli consisted of brief biphasic pulses (200 $\mu \mathrm{s}$, intensities 5-80 V) triggered by an analog stimulus isolation unit (BSI-950, Dagan Corporation, USA). Stimulation intensities between 5-80 V were used in these experiments. Threshold for synaptic responses was usually between 10-20 V. For the analysis of input number the stimulation intensity was gradually increased in increments of 2-10 V. Inhibitory (glycinergic and GABAergic) or excitatory (AMPA and NMDA) postsynaptic currents were isolated by applying $1 \mu \mathrm{M}$ Strychnine and $10 \mu \mathrm{M}$ SR95531 or $10 \mu \mathrm{M}$ DNQX and $25 \mu \mathrm{M}$ DL-AP5 to the bath, respectively.

All agents were purchased from Sigma-Aldrich (Seelze, Germany) and Biotrend (Köln, Germany) unless otherwise indicated.

\section{Data Acquisition and Analysis}

For all electrophysiological measurements, stimulus generation and recordings were acquired using pCLAMP (Axon Instruments, USA). Current and voltage clamp signals were 
low-pass filtered at $10 \mathrm{kHz}$ with a Bessel filter, sampled at a rate of $20-50 \mathrm{kHz}$ and analyzed using Clampfit 10.0 (Axon Instruments, USA) and customized macros in IGOR Pro (Wavemetrics, USA). A junction potential of $-10.5 \mathrm{mV}$ was subtracted for recordings using a K-Gluconate solution.

The resting membrane potential was calculated as the mean value of the first $80 \mathrm{~ms}$ before hyperpolarizing or depolarizing steps were applied. Membrane time constant and input resistance were evaluated from the voltage response to a current injection of $-100 \mathrm{pA}$. Membrane time constants were calculated by fitting single-exponential functions to the voltage traces. Input resistance was measured at the peak hyperpolarization according to Ohm's law. For analyzing the active membrane properties, the action potential or the train of action potentials elicited by the lowest current injection was used to measure current threshold, voltage threshold, action potential amplitude, half-width, and latency.

Half-maximal activation of $\mathrm{I}_{\mathrm{h}}$ was computed from tail currents. Tail current amplitudes were measured $20 \mathrm{~ms}$ after the termination of the hyperpolarizing voltage steps. These amplitudes were normalized to the maximal amplitude of each neuron. Values were fitted to a Boltzmann function to obtain the half-maximal activation voltage $\mathrm{V}_{1 / 2}$.

During synaptic stimulation experiments the number of input fibers was determined by visually identifying groups of similar excitatory post-synaptic current (EPSC) and inhibitory postsynaptic current (IPSC) amplitudes while stimulation intensities were gradually increased from 10-80 V. Each group presumably reflects the activation of an individual input fiber (Kim and Kandler, 2003; Walcher et al., 2011).

$10-90 \%$ rise time and tau decay (fitting of a singleexponential function) of synaptic currents were assessed from responses evoked by minimal stimulation. The minimal stimulation strength is defined as stimulation strength where a stepwise increase of $2 \mathrm{~V}$ results in the first measureable post synaptic current (PSC). To estimate short-term plastic changes of excitatory and inhibitory inputs to repetitive stimulations (1-300 $\mathrm{Hz}, 15$ pulses, 10 repetitions), PSC amplitudes in the trains were normalized to the first amplitude. For calculating net amplitudes at high frequencies, a decay function was fitted to the preceding current pulse and set as new baseline. The amplitude of the respective pulse was measured as a subtraction of the maximum and the new baseline. From these normalized depression curves steady state depression was obtained by the mean of the last three PSC amplitudes.

Results are shown as mean \pm standard error of the mean. Statistical significance was either calculated by the unpaired student's $t$-test or single factor ANOVA followed by a Scheffé's post hoc test in Excel (Microsoft) or Igor Pro (Wavemetrics, USA) with statistical thresholds of ${ }^{*} p<0.0,{ }^{* *} p<0.01$ and ${ }^{* * *} p<0.001$. Kruskal-Wallis test was used, as indicated, for nonparametric distributions.

\section{Immunohistochemistry}

Animals were anesthetized by an intraperitoneal injection of Fentanyl (Janssen-Cilag), Medetomidin (Ratiopharm) and
Metformin (Pfizer). They were perfused transcardially with $0.1 \mathrm{M}$ phosphate buffer (PB; $\mathrm{pH} 7.4$ ) for $3 \mathrm{~min}$ followed by Paraformaldehyde (Carl Roth, Karlsruhe, Germany; PFA; $4 \%$ in $0,1 \mathrm{M} \mathrm{PB} ; \mathrm{pH} 7.4$ ) for $15 \mathrm{~min}$. Immediately after perfusion, brains were removed and post-fixed overnight in $4 \%$ PFA at $4^{\circ} \mathrm{C}$. Brains were thoroughly washed at room temperature with $0.1 \mathrm{M}$ phosphate buffered saline (PBS; $\mathrm{pH}$ 7.4). Coronal brain sections of $50 \mu \mathrm{m}$ were obtained using a vibratome (VT1200, Leica, Germany). Sections containing the VNLL were collected. Unspecific binding was blocked incubating the sections in solution containing 10\% normal donkey serum (NDS; GeneTex, USA), 0.2\% Triton X-100 (Carl Roth, Karlsruhe, Germany) and 0.1 M PBS, for an hour at room temperature. Slices were subsequently incubated overnight in the primary antibody sera containing mouse $\alpha-\mathrm{HCN} 1$ (NeuroMab; dilution 1:1000), rabbit $\alpha$-VGluT1 (Synaptic Systems; dilution 1:500), chicken $\alpha$-MAP2 (Neuromics; dilution 1:1000), goat $\alpha$-Calretinin (Millipore; dilution 1:4000), 3\% NDS, and 0.2\% Triton X-100, in 0.1 M PBS.

Slices were washed in $0.1 \mathrm{M}$ PBS and incubated for $2 \mathrm{~h}$ at room temperature in secondary antibody sera containing $3 \%$ NDS, $0.2 \%$ Triton X-100, Alexa 488 donkey $\alpha$-mouse (life technologies; dil. 1:250), Alexa 555 donkey $\alpha$-rabbit (life technologies; dilution 1:500), Cy3 donkey $\alpha$-goat (Dianova; dilution 1:300), and Alexa 647 donkey $\alpha$-chicken (Dianova; dilution 1:300). Negative controls were obtained by omitting the primary antibody. Sections were washed several times in 0.1 M PBS, mounted and covered with homemade anti-fading mounting media (Indig et al., 1997). All sections were analyzed with a Leica SP8 confocal laser scanning microscope. Three laser lines for visualization were used. The fluorochromes Alexa 488, Alexa 555 and Alexa 647 were excited by an Ar-Ion-Laser line of $488 \mathrm{~nm}$, the single DPSS $561 \mathrm{~nm}$ laser line and the $\mathrm{HeNe}$ single laser line of $633 \mathrm{~nm}$, respectively. Stacks of confocal images were further analyzed and edited with Image $(\mathrm{NIH}$, USA).

\section{RESULTS}

\section{Intrinsic Properties Differ between Neurons of the Dorsal and the Ventral Parts of the VNLL}

Neuron types are largely determined by their biophysical membrane properties which are dependent on the composition of ion channels in the membranes. To find out whether there is a systematic distribution of neuronal cell types across the VNLL we analyzed intrinsic membrane properties with respect to their location within the VNLL in a brain slice preparation of young adult mice (P22/23).

The VNLL is an elongated structure with a ventral and a dorsal area that are enriched with neurons (Figure 1A). In ventral VNLL (vVNLL) neurons, depolarizing current injections elicited an onset-type firing pattern in 34 out of 37 (Figure 1B). In the remaining three neurons depolarization induced an onset burst firing pattern (data not shown). In all vVNLL neurons, a negative current injection led to a large hyperpolarization 
followed by a slowly depolarizing voltage sag. In the dorsal part of the VNLL (dVNLL), positive current injections induced either an onset-type firing pattern $(n=19)$ or a sustained firing pattern $(n=9$; Figure 1C). In onset-type firing neurons negative current injections induced a small hyperpolarization which repolarized rapidly. In sustained-firing neurons, the hyperpolarization was more pronounced with a prominent voltage sag. Onset-type firing neurons of the vVNLL and dVNLL significantly differed in their resting membrane potential, their membrane time constant and their input resistance (Figures 1D-F; Table 1). Onset-type firing neurons of the dVNLL were fastest and exhibited the smallest peak input resistance suggesting different subpopulation of onset-type firing neurons in the vVNLL and dVNLL.

We next analyzed active membrane properties of the different VNLL cell types at the first supra-threshold response (Figures 2A,B). Onset-firing neurons of the ventral and the dorsal regions differed significantly in current and voltage threshold, whereas sustained firing neurons of the dorsal part displayed differences to onset-type firing neurons of both regions regarding AP amplitude and AP latency (Figures $2 \mathrm{C}-\mathbf{H}$; Table 1). Sustained-firing neurons of the dVNLL had the lowest current threshold, largest AP amplitudes and latencies, whereas onset-type firing cells of the same region had the highest current threshold of all neurons measured (Figures 2D,F,H; Table 1). AP half-width did not differ between vVNLL and dVNLL neurons (Figure 2G; Table 1).

We conclude that the dVNLL consists of two different subpopulations of neurons with distinct intrinsic membrane properties. The population of vVNLL neurons, however, seems to be fairly uniform regarding their intrinsic properties.

\section{Dorsal VNLL Neurons have Large Hyperpolarization-Activated Currents $\left(l_{h}\right)$ Compared to Ventral VNLL Neurons}

Differences in the resting membrane potential and in the voltage sag induced by hyperpolarization between dorsal and ventral VNLL neurons indicate a differential distribution of
HCN channels between the two parts. Our electrophysiological measurements of $I_{h}$ in different parts of the VNLL revealed only small $\mathrm{I}_{\mathrm{h}}$ amplitudes in the vVNLL (Figure 3A), whereas $\mathrm{I}_{\mathrm{h}}$ amplitudes were large in dVNLL neurons (Figure 3B). Consequently, $I_{h}$ density was significantly larger in dVNLL neurons as compared to vVNLL neurons (at $-110.5 \mathrm{mV}$ vVNLL: $-24.0 \pm 3.4 \mathrm{pA} / \mathrm{pF}, n=13$; dVNLL: $-84.3 \pm 12.9 \mathrm{pA} / \mathrm{pF}$, $n=12$; student's unpaired $t$-test, $p=0.0006$; Figures $3 \mathrm{C}, \mathrm{D}$ ). Half-maximal activation voltage for $\mathrm{HCN}$ channels in the dVNLL was $-88.3 \pm 3.0 \mathrm{mV}(n=10$, Figure $3 \mathrm{E})$, whereas tail-currents evoked in vVNLL neurons were too small to be reliably analyzed. Consistently, immunostainings against the HCN1 subunit showed that this subunit is strongly expressed in the dVNLL, whereas neurons in the vVNLL are mostly HCN1 negative (Figures 3F1-F3) indicating that HCN1 channels are the predominant isoform in dVNLL neurons.

Neurons in the vVNLL all have onset-type firing properties, little $\mathrm{I}_{\mathrm{h}}$ and relatively large input resistance during hyperpolarization. In contrast, all dVNLL neurons have large $\mathrm{I}_{\mathrm{h}}$ currents and small input resistance which results in short membrane time constants during hyperpolarization.

\section{Lateral vVNLL Neurons have Extremely Large Excitatory Postsynaptic Currents}

We were further interested to what extent the excitatory and inhibitory input properties differed between the two regions. Inputs were stimulated in the fiber bundle about $50 \mu \mathrm{m}$ ventral to the recorded neuron and EPSCs were isolated pharmacologically. Stimulation intensity was systematically increased to estimate the number of input fibers (=step number; see "Materials and Methods" Section). During these experiments we noticed that the vVNLL can be subdivided into two neuronal population based on the estimated number and the peak amplitude of excitatory inputs the neurons received. A large population of vVNLL neurons that were located in a cluster laterally to the fiber bundle all exhibited an all-or-none EPSC response with extremely large amplitudes irrespective of stimulation

TABLE 1 | Summary of membrane properties of dVNLL and vVNLL neurons.

\begin{tabular}{|c|c|c|c|c|}
\hline & \multirow{2}{*}{$\begin{array}{c}\text { Ventral } \\
\text { Onset }(n=34)\end{array}$} & \multicolumn{2}{|c|}{ Dorsal } & \multirow[b]{2}{*}{$p$} \\
\hline & & Onset $(n=19)$ & Sustained $(n=9)$ & \\
\hline \multicolumn{5}{|l|}{ Passive membrane properties } \\
\hline Input resistance $(\mathrm{M} \Omega)$ & $151.7 \pm 43.8$ & $43.8 \pm 6.3$ & $111.4 \pm 19.4$ & $* * *^{\mathrm{C}}$ \\
\hline Membrane time constant (ms) & $5.36 \pm 0.51$ & $2.41 \pm 0.29$ & $7.31 \pm 1.30$ & $* *^{\mathrm{c}}, * * *^{\mathrm{a}}$ \\
\hline \multicolumn{5}{|l|}{ Active membrane properties } \\
\hline AP amplitude (mV) & $58.7 \pm 0.9$ & $56.6 \pm 2.0$ & $66.7 \pm 2.6$ & $*^{\mathrm{b},} * *^{\mathrm{a}}$ \\
\hline AP half-width (ms) & $0.47 \pm 0.03$ & $0.46 \pm 0.03$ & $0.39 \pm 0.02$ & n.s \\
\hline AP latency (ms) & $2.0 \pm 0.1$ & $2.5 \pm 0.3$ & $4.0 \pm 0.4$ & $* *^{\mathrm{a}}, * * *^{\mathrm{b}}$ \\
\hline
\end{tabular}




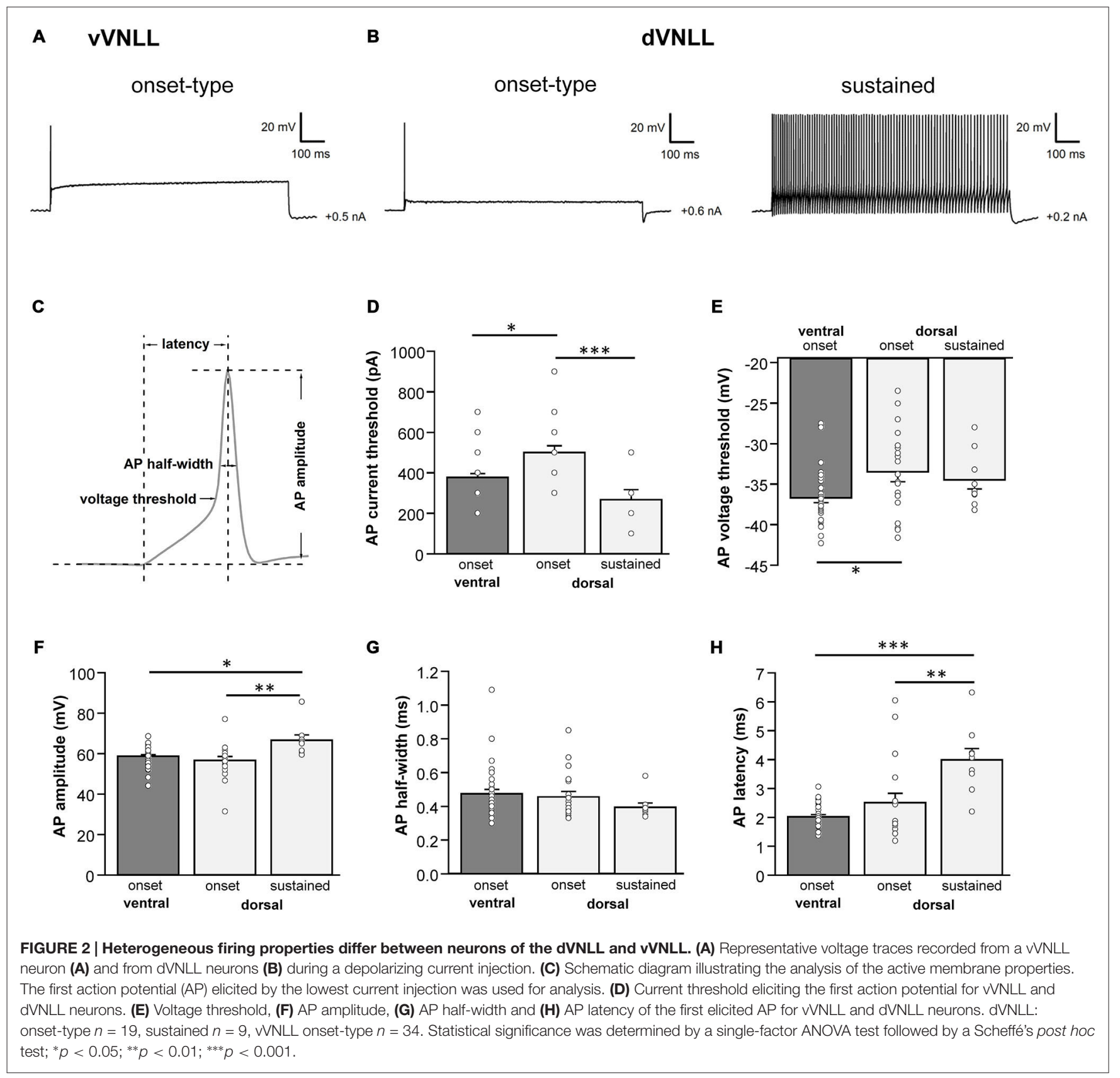

strength (Figures 4A1,A2 left). This was different in the ventromedial part of the VNLL where neurons are scattered within the fiber bundle. There, EPSC amplitude gradually increased with rising stimulation strength which indicates a convergence of several input fibers (Figures 4A1,A2 right). Step number and maximally evoked EPSC amplitude differed significantly between ventrolateral and ventromedial VNLL neurons (Figures 4C,D; Table 2). Excitatory input properties of neurons in the dVNLL very much resembled those found in the medial neurons of the vVNLL (Figures 4B1,B2; Table 2). Moreover, $10-90 \%$ rise time and tau decay time of EPSCs were much faster in ventrolateral compared to ventromedial and dorsal neurons (Figure 4E; Table 2). We reanalyzed membrane properties according to their mediolateral location in the vVNLL. Nevertheless, membrane properties were very similar in medial and lateral VNLL neurons despite the profound differences in excitatory input characteristics (resting membrane potential: ventrolateral $-64.4 \pm 0.4 \mathrm{mV}, n=$ 29 , ventromedial $-65.2 \pm 0.8 \mathrm{mV}, n=5$; input resistance: $-150.17 .3 \mathrm{M} \Omega, n=29$; ventromedial $-158.8 \pm 26.5 \mathrm{M} \Omega$, $n=5$; membrane time constant: ventrolateral $5.3 \pm 0.58$ $\mathrm{ms}, n=29$; ventromedial $5.71 \pm 1.11 \mathrm{~ms}, n=5$, data not shown). These physiological findings were corroborated by a differential distribution of VGluT1 and calretinin positive 
A vVNLL

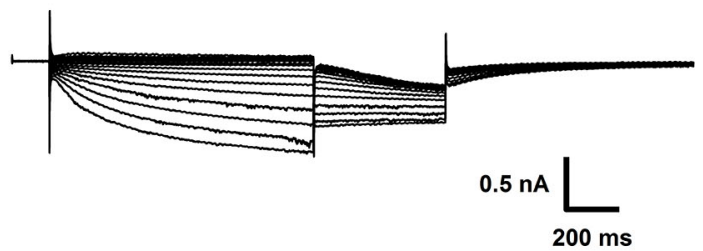

$-70 \mathrm{mV}$

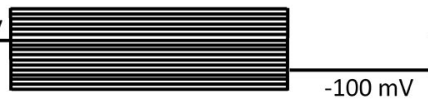

C
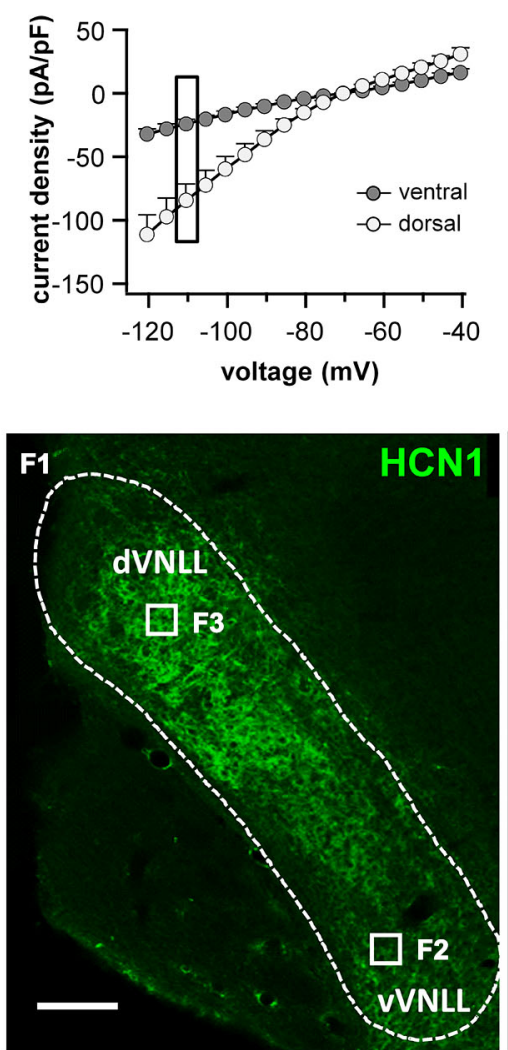

B

dVNLL

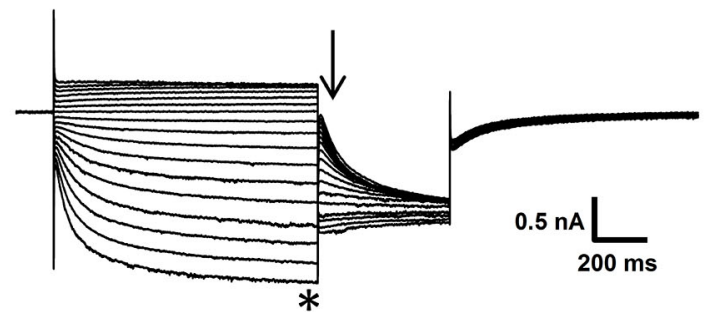

E

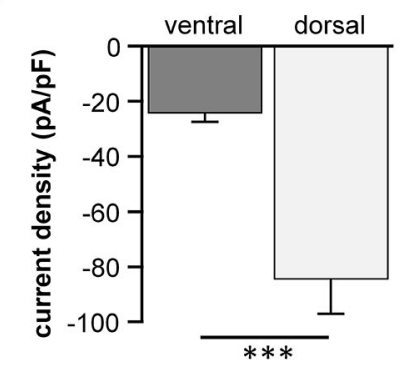

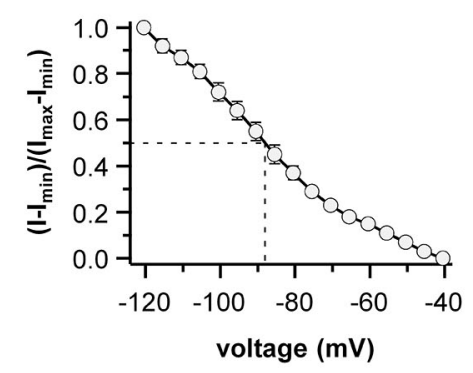
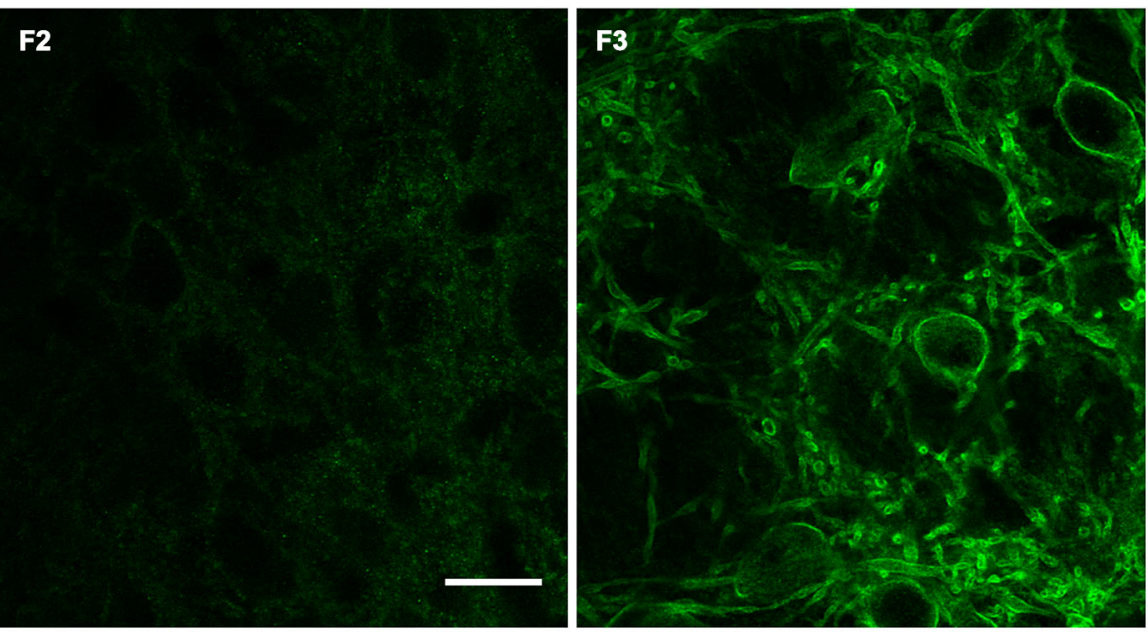

FIGURE 3 | DVNLL neurons have larger hyperpolarization-activated currents ( $\mathbf{I}_{\mathbf{h}}$ ) and stronger HCN1 immunoreactivity. Representative current traces recorded from VVNLL neurons (A) and dVNLL neurons (B) to voltage steps ranging from -40 to $-120 \mathrm{mV}$ in $5 \mathrm{mV}$ step increment. Each voltage step lasted for $1 \mathrm{~s}$ and was followed by a voltage step to $-100 \mathrm{mV}$ for $0.5 \mathrm{~s}$ to elicit the tail current to determine the voltage dependence of $\mathrm{I}_{\mathrm{h}}$ activation. Holding potential was set to $-70 \mathrm{mV}$. (C) Current density-voltage relationship for dVNLL and VVNLL neurons. Current amplitudes were measured at steady-state (indicated by the asterisk in B). Rectangular labeling is exemplified in (D). (D) Current density at $-110 \mathrm{mV}$ for VVNLL and dVNLL neurons are significantly different. dVNLL: $n=12$, vVNLL $n=13$. Statistical significance was determined by an unpaired student's $t$-test; ${ }^{* * *} p<0.001$. (E) The voltage-dependence of $I_{h}$ activation was determined from the tail current $20 \mathrm{~ms}$ after the end of the voltage steps (indicated by the arrow in B). Values were fitted with a Boltzmann function. Half-maximal activation voltage for neurons was at around $-88 \mathrm{mV}(n=10)$. Half-maximal activation voltage of VVNLL neurons is not shown since almost no current is present. Data are obtained from eight C57/Bl6J mice. (F) Confocal images of HCN1 immunostaining in the VNLL. (F1) Overview illustrating a gradient of HCN1 staining in the VNLL. (F2) High magnification of a region within the VVNLL reveals that HCN1 staining is almost absent. (F3) High magnification of a region within the dVNLL shows strong HCN1 staining confined to the membrane. Scale bar: (F1) $200 \mu \mathrm{m}$; (F2) $20 \mu \mathrm{m}$.

presynaptic structures in different parts of the VNLL. Whereas in the ventrolateral VNLL a distinct perisomatic VGluT1-positive ring, that co-localized with calretinin, was detected around the somata (Figure 4F1), in both the ventromedial VNLL and the dorsal VNLL VGluT1-staining appeared scattered in the neuropil, presumably representing dendritic inputs 


\begin{tabular}{|c|c|c|c|c|}
\hline & \multicolumn{2}{|c|}{ Ventral } & \multirow[t]{2}{*}{ Dorsal } & \multirow[b]{2}{*}{$p$} \\
\hline & Lateral & Medial & & \\
\hline \multicolumn{5}{|c|}{ Excitatory postsynaptic currents } \\
\hline Step number & $1.13 \pm 0.13(8)$ & $3.00 \pm 0.46(8)$ & $3.85 \pm 0.63(7)$ & $*^{\mathrm{a},} * *^{\mathrm{b}}$ \\
\hline Max. EPSC amplitude (nA) & $11.47 \pm 1.48(8)$ & $2.39 \pm 0.45(8)$ & $1.66 \pm 0.76(7)$ & $* * *^{\mathrm{a}, \mathrm{b}}$ \\
\hline $10-90 \%$ rise time (ms) & $0.18 \pm 0.01(9)$ & $0.40 \pm 0.08(8)$ & $0.44 \pm 0.04(14)$ & $*^{\mathrm{a},} * *^{\mathrm{b}}$ \\
\hline Tau decay (ms) & $0.31 \pm 0.01(9)$ & $1.24 \pm 0.37(8)$ & $1.63 \pm 0.24(14)$ & $* *^{\mathrm{b}}$ \\
\hline \multicolumn{5}{|c|}{ Inhibitory postsynaptic currents } \\
\hline Step number & $2.70 \pm 0.26(10)$ & $2.38 \pm 0.26(8)$ & $2.58 \pm 0.36(12)$ & \\
\hline Max. EPSC amplitude (nA) & $5.95 \pm 1.03(10)$ & $2.80 \pm 1.08(8)$ & $2.80 \pm 0.81(12)$ & \\
\hline $10-90 \%$ rise time $(\mathrm{ms})$ & $0.37 \pm 0.02(16)$ & $0.49 \pm 0.07(10)$ & $0.48 \pm 0.07(14)$ & \\
\hline Tau decay (ms) & $2.47 \pm 0.17(16)$ & $1.88 \pm 0.11(10)$ & $3.06 \pm 0.68(14)$ & \\
\hline
\end{tabular}

Values are mean \pm SEM. The level of significance between ventral and dorsal was determined using Scheffé's post hoc test following a single-factor ANOVA test or Games Howell post hoc test following Kruskal-Wallis for non-parametric distributions $\left({ }^{*} p<0.05,{ }^{* *} p<0.01,{ }^{* * *} p<0.001\right)$. Numbers of neurons are given in brackets. a Significant differences between lateral and medial neurons of the VVNLL. ${ }^{b}$ Significant differences between dVNLL and lateral VVNLL neurons.

(Figures 4F2,F3). This implies that neurons in the ventrolateral VNLL receive one large excitatory synapse with calyx-like properties similar to MNTB neurons. In contrast, medioventral and dorsal VNLL neurons integrate several excitatory inputs with smaller amplitudes that terminate predominantly on the dendrites.

\section{Inhibitory Postsynaptic Currents are Similar in all vVNLL Neurons}

We were also interested whether inhibitory input characteristics differed between the various subregions of the VNLL (Figures 5A1,A2,B1,B2). In all three regions of the VNLL the number of inhibitory inputs was very similar (Figure $5 \mathrm{C}$; Table 2). In accordance with the excitatory input strength, maximal inhibitory input amplitude was significantly larger in ventrolateral neurons compared to other VNLL areas (Figure 5D; Table 2). The temporal characteristics of inhibitory inputs were very similar in all parts of the VNLL (Figure 5E; Table 2).

Taken together, we show that VNLL neurons exhibit similar inhibitory synaptic responses which suggest a common origin of these inputs.

\section{Synaptic Short-Term Plasticity of Excitatory and Inhibitory Inputs is Similar in vVNLL and dVNLL Neurons}

We further characterized short-term plasticity (STP) of excitatory and inhibitory synapses in vVNLL and dVNLL neurons (15 stimuli at 1, 50, 100, 200 and $300 \mathrm{~Hz}$ ). All excitatory and inhibitory inputs to VNLL neurons showed pronounced synaptic short-term depression that depended on the stimulation frequency (Figures 6A-D). In order to compare synaptic short-term depression of vVNLL and dVNLL neurons the steady state depression as a function of stimulus frequency is shown in Figures 6E,F. For both EPSCs and IPSCs steady state depression did not differ between vVNLL and dVNLL neurons for almost all stimulus frequencies. Only for stimulation frequencies of $100 \mathrm{~Hz}$ steady state depression of EPSCs was significantly larger for vVNLL compared to dVNLL neurons (vVNLL: $50 \pm 7 \%$; dVNLL: $32 \pm 5 \%,{ }^{*} p<$ 0.05 , unpaired student's $t$-test). This is a further indication that excitatory inputs differ between the ventrolateral region and the other regions of the VNLL, whereas inhibitory input properties are very similar amongst neurons in the entire VNLL.

\section{Neurons in the Dorsal and the Ventral VNLL are all Inhibitory}

To test whether the neurons we recorded from were inhibitory and therefore part of the VNLL, we recorded from neurons of VGAT-ChR2-YFP ${ }^{+}$mice. We tested the transmitter phenotype of neurons in both the vVNLL and dVNLL by applying short pulses of white light onto the slice while recording the membrane potential. All VNLL neurons, in the dorsal and ventral part, showed a pronounced depolarization in response to the light stimulus (Figures 7A,B,D). The amplitude of the depolarization varied dependent on the input resistance of the neuron (vVNLL: $3.8 \pm 0.8 \mathrm{mV}, n=6$; dVNLL: onset-type $2.2 \pm 0.4 \mathrm{mV}, n=9$, sustained $8.8 \pm 2.3$ $\mathrm{mV}, n=6$; INLL: $-1.4 \pm 0.4 \mathrm{mV}, n=8$; Figure 7D). A population of small neurons in between our region of interest (dVNLL) and the DNLL did not depolarize during light application (Figures 7C,D). We therefore consider this population as glutamatergic and according to Ito et al. (2011) as INLL neurons, whereas our sample of neurons show an inhibitory neurotransmitter type and are thus located within the VNLL.

\section{DISCUSSION}

The present study demonstrates several distinct subpopulations of neurons in the dorsal and ventral parts of the VNLL. One distinct subpopulation of neurons, located in the ventrolateral part of the VNLL, displayed an onset-type firing pattern and only little $\mathrm{I}_{\mathrm{h}}$. These neurons received mostly one large calyx-like 
A1

lateral

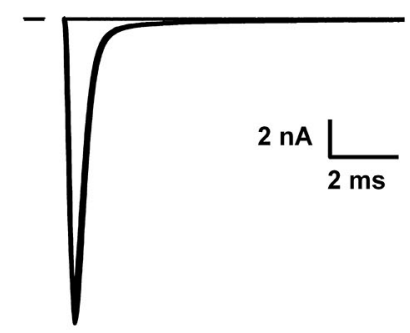

A2

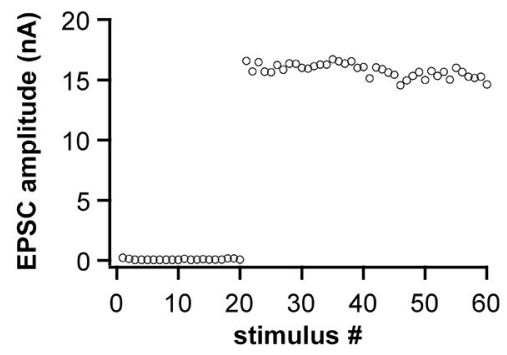

$20 \mathrm{~V}$ stimulus strength $70 \mathrm{~V}$

C

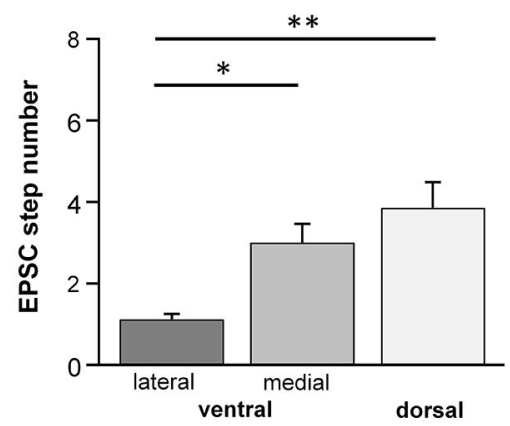

VVNLL
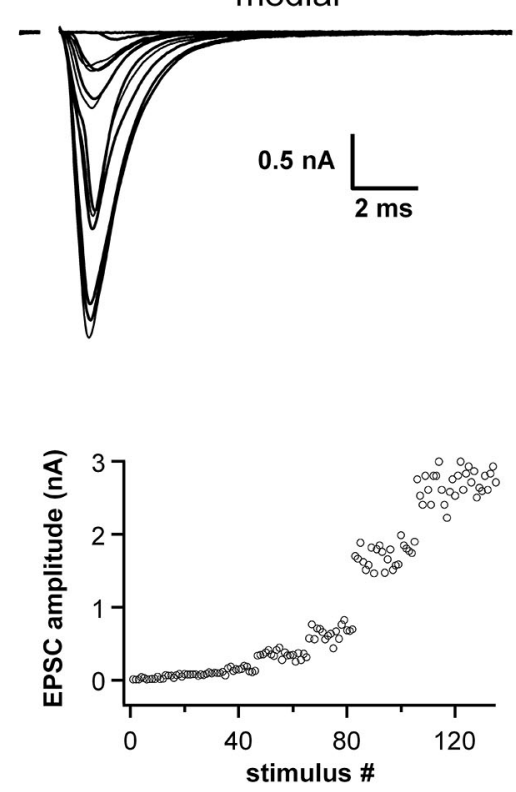

$10 \mathrm{~V}$ stimulus strength $80 \mathrm{~V}$

D

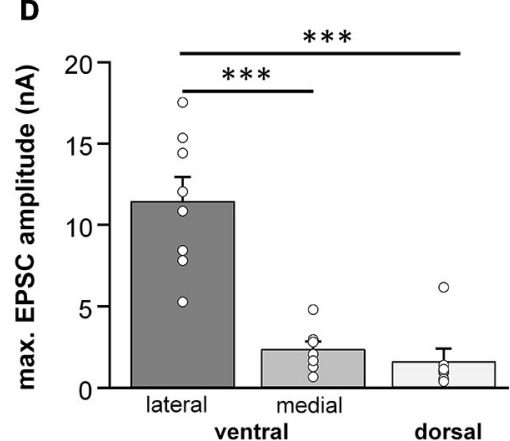

B1 dVNLL

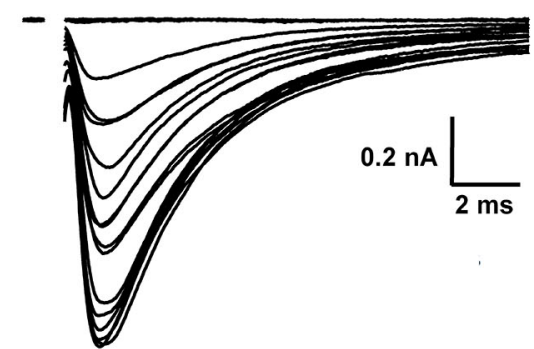

B2

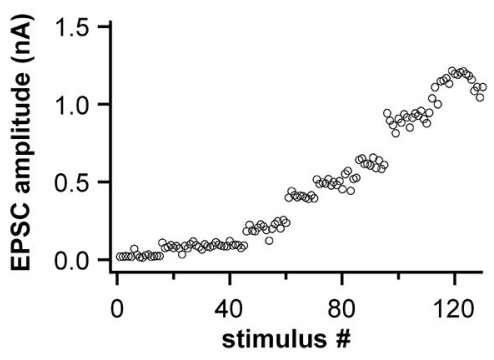

$3 \mathrm{~V}$ stimulus strength $70 \mathrm{~V}$

E

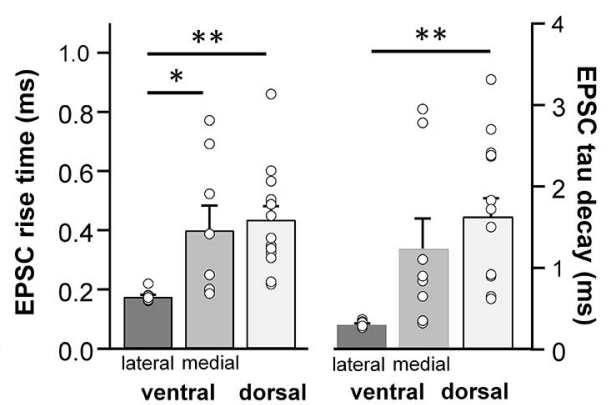

F
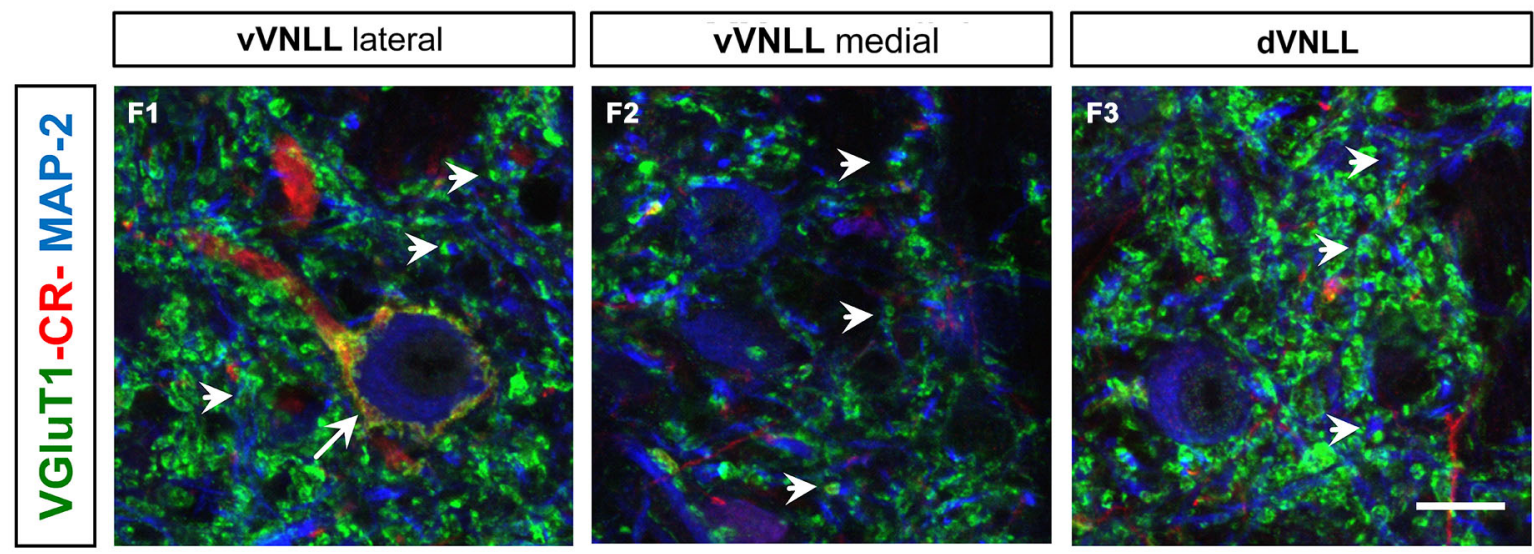

FIGURE 4 | Excitatory postsynaptic currents (EPSCs) are larger and the excitatory inputs are stronger in vVNLL neurons. (A1) Representative EPSC traces of ventrolateral (left) and ventromedial (right) VNLL neurons. Responses were evoked by increasing stimulation strength. One strong input was found in most ventrolateral neurons, whereas medial neurons showed multiple inputs. All traces represent the average of at least three consecutive recordings to the same stimulus strength. (A2) EPSC amplitude as a function of stimulus number recorded from ventrolateral and ventromedial vVNLL neurons shown in (A1). (B1) Representative 


\section{FIGURE 4 | Continued}

EPSC traces of dVNLL neurons. Responses were evoked with increasing stimulation strength. This neuron shows seven distinct current steps. Note the considerably smaller maximal amplitude compared to ventrolateral VNLL neurons. All traces represent the average of at least three consecutive recordings to the same stimulus strength. (B2) EPSC amplitude as a function of stimulus number recorded from the dVNLL neuron shown in (B1). (C) Number of EPSC steps evoked by increasing stimulation strength in VVNLL and dVNLL neurons. Ventrolateral VNLL neurons generally receive one, maximally two excitatory inputs whereas ventromedial and dVNLL neurons receive one to seven inputs. VVNLL: lateral $n=8$, medial $n=8$; dVNLL $n=7$. (D) Maximal EPSC amplitude is significantly larger in ventrolateral VVNLL neurons. VVNLL: lateral $n=8$, medial $n=8$; $\mathrm{dVNLL} n=7$. (E) Rise time and tau decay time were measured in VVNLL and dVNLL neurons applying a minimal stimulation paradigm. Ventrolateral neurons are significantly faster than dVNLL neurons. VVNLL: lateral: $n=9$; medial: $n=8$; dVNLL: $n=14$. Statistical significance was determined by a single-factor ANOVA test followed by a Scheffé's post hoc test; ${ }^{*} p<0.05,{ }^{* *} p<0.01,{ }^{* * *} p<0.001$. Data are obtained from $21 \mathrm{C} 57 / \mathrm{Bl6J}$ mice. (F) Confocal images depicting VGlut1, calretinin (CR) and MAP-2 immunofluorescence in the lateral VVNLL (F1), the medial VVNLL (F2) and the dVNLL (F3). Note a VGluT1 and Calretinin positive ring only found around lateral VVNLL neurons (F1: long arrow) whereas punctate VGluT1 staining in the neuropil is widely distributed along the VNLL (F1-F3). Scale bar: $10 \mu \mathrm{m}$.

excitatory input, multiple large inhibitory inputs and were densely packed in a cluster lateral to the fiber bundle. A second cell type, located in the ventromedial part of the VNLL, was scattered within the fiber bundle. Although these neurons exhibited similar firing and intrinsic properties compared to ventrolateral neurons, they received several and much weaker excitatory inputs. In the dVNLL, neurons with two different firing patterns are intermingled. Neurons showed either a single spike at the onset of the response or a sustained firing pattern. Moreover, all dorsal neurons exhibited large $I_{h}$. The onsettype firing neurons in the dorsal part clearly differed from the onset-type firing neurons in the ventral part of the VNLL in terms of their intrinsic properties. Ventromedial VNLL neurons that were intermingled within the lemniscal fiber tract had intrinsic properties similar to the ventrolateral neurons and synaptic properties that resembled those of neurons in the dVNLL. All neuron types in the dorsal and ventral VNLL depolarized by light stimulation in a VGAT-Channelrhodopsin mouse model where channelrhodopsin is expressed under the promoter of VGAT (Zhao et al., 2011). Since in this mouse model only inhibitory neurons depolarize by light stimulation our sample neurons were inhibitory and were rather part of the VNLL and not the INLL (Saint Marie et al., 1997; Ito et al., 2011).

\section{The Ventral Part of the VNLL Contains a Cluster of Neurons with Calyx-Like Synaptic Inputs}

This study describes a distinct population of neurons in the vVNLL with biophysical membrane properties that are very similar to those found in neurons of the MNTB (Brew and Forsythe, 1995; Hassfurth et al., 2009). Both of these neuron populations have a typical onset-type response pattern, little $\mathrm{I}_{\mathrm{h}}$ and a lack of HCN1 immunoreactivity. Previously, similar onset-type firing neurons have been described in the ventral part of the rat VNLL which were correlated with a bushy-cell like morphology (Wu, 1999; Zhao and $\mathrm{Wu}, 2001)$. However, only around $25 \%$ of the neurons studied belonged to this neuron-type. Explanation for this relatively low fraction of onset neurons might be the different developmental time frames studied, variations in recording sites (dorsal vs. ventral) and the different recording techniques employed. A very recent study of gerbil VNLL neurons with a Calyx-like input demonstrated a developmental change from a sustained to an onset-type firing pattern within the first week after hearing onset (Berger et al., 2014; Franzen et al., 2015). However, neither the spatial distribution of neurons within the VNLL nor the existence of other cell types was discussed in this study.

Our data show that these neurons receive one large calyx-like excitatory input similar in amplitude and temporal properties to the Calyx of Held synapse in the MNTB (Borst and Soria van Hoeve, 2012). Moreover, these neurons are surrounded by a VGluT1 and calretinin positive ring, which suggests that this input originates from octopus cells of the contralateral VCN (Adams, 1997; Schofield and Cant, 1997). Similar calyx-like synaptic structures with comparable physiological characteristics were recently described in the gerbil VNLL (Berger et al., 2014). However, in this study VNLL neurons were usually contacted by two large excitatory synaptic inputs. Whether this is a species specific adaptation or whether a further developmental reduction of inputs occurs after P12, when these experiments were performed, remains unexplored. Anatomical studies in other mammals identified a subdivision within the ventral VNLL with one densely and fairly uniform arrangement of neurons with spherical or globular bushy cell morphology (Willard and Ryugo, 1983; Vater and Feng, 1990; Adams, 1997; Schofield and Cant, 1997; Benson and Cant, 2008), which suggests that this neuron type is commonly found in all mammalian species. In fact, an anatomical study suggests that in humans this area is even hypertrophied (Adams, 1997). Interestingly, neurons in the ventral VNLL, that were not located in this lateral cluster but were intermingled within the fiber tract of the lateral lemniscus, received several excitatory inputs with much smaller amplitude similar to dVNLL neurons, while intrinsic properties were indistinguishable from the ventrolateral VNLL neurons. Further experiments are needed to determine whether input origin and response properties of these neurons are more similar to ventrolateral or dorsal VNLL neurons.

\section{Neurons in the Dorsal Part of the VNLL have Two different Firing Patterns and Large $I_{h}$}

Dorsal VNLL neurons responded with two different firing patterns to depolarizing current injections. Whereas one population of neurons had the typical onset-type response similar to principal cells in the lateral and medial superior olive with low input resistance and large $\mathrm{I}_{\mathrm{h}}$ (Mathews et al., 2010; Walcher et al., 2011), the other neuronal population fired throughout the depolarizing stimulus and 


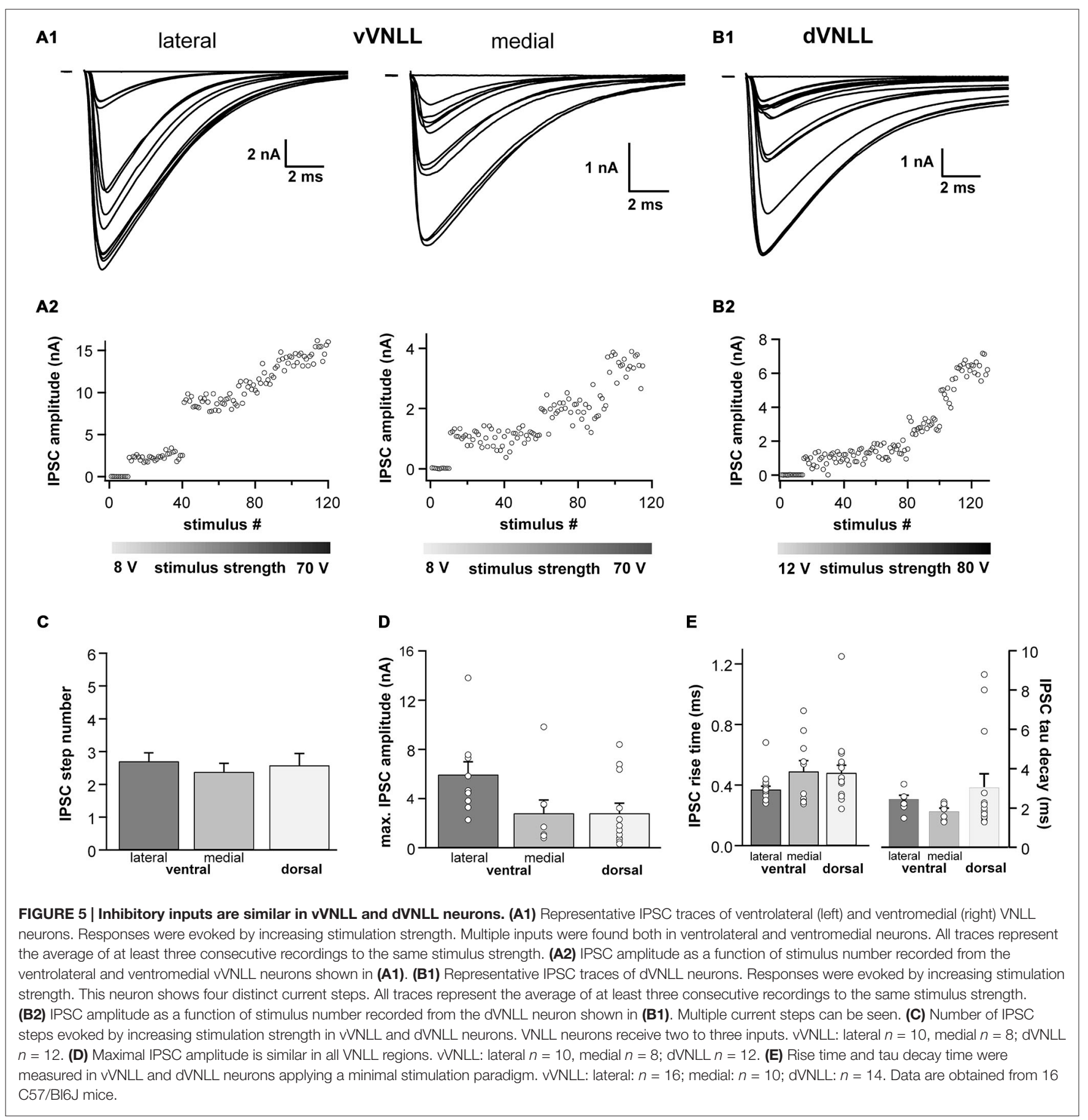

with increasing frequency for stronger depolarization. This suggests a differential distribution of K-channels, especially the low-threshold K-channels of the Kv1 family, that are usually abundant in neurons with an onset-type firing pattern (BarnesDavies et al., 2004; Mathews et al., 2010). Morphologically, two different neuron types are present in the dVNLL. The most frequent one are multipolar cells that are densely packed in the most dorsal part of the VNLL in most species (Schofield and Cant, 1997; Benson and Cant, 2008). Another less frequent type are the giant cells (Schofield and Cant, 1997; Benson and Cant, 2008). Whether this morphological cell type corresponds to our onset or sustained firing type is unclear, but could be determined by studying K-channel distribution in the VNLL.

In the dVNLL all neurons had relatively large $I_{h}$ by visually identifying groups and displayed intense immunostaining against the HCN1 channel. Among other HCN subtypes the HCN1 subunit is the one with the fastest activation time constant and the most depolarized half-maximal activation of 


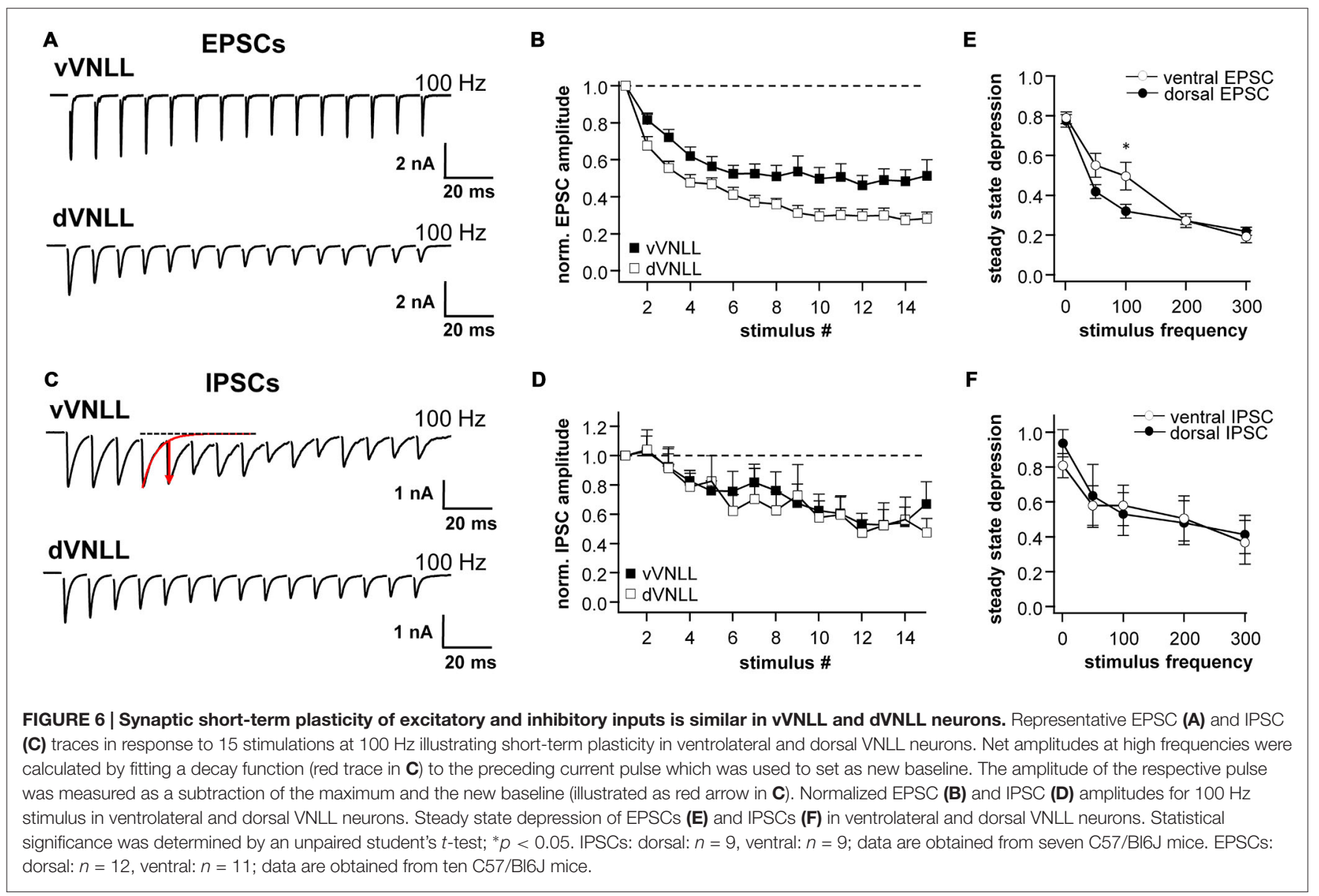

$I_{h}$ (Wahl-Schott and Biel, 2009). Therefore, neurons with large HCN1 mediated currents integrate excitatory and inhibitory inputs with a high temporal resolution and little summation (Khurana et al., 2012; Baumann et al., 2013). Consistently, most of the neurons received excitatory and inhibitory inputs with fast synaptic time constants. These fast synaptic currents together with the rapid membrane time constants suggest that these neurons might contribute to temporal sound pattern analysis by integrating their inputs with high temporal precision as suggested for VNLL neurons (Nayagam et al., 2005; Yavuzoglu et al., 2011; Recio-Spinoso and Joris, 2014).

\section{Dorsal and Ventral VNLL Neurons are Inhibitory}

Several studies have shown that a population of neurons in the most dorsal VNLL differs in terms of input projection, binaurality and neurotransmitter type compared to more ventral VNLL neurons. These dorsal VNLL neurons mostly express excitatory markers whereas most neurons in ventral VNLL are either GABAergic, glycinergic or co-express both inhibitory transmitters (Saint Marie et al., 1997; Riquelme et al., 2001; Ito et al., 2011). The very dorsal VNLL neurons also receive prominent projections from binaural nuclei of the superior olive (Kelly et al., 2009) which are mostly absent in the ventral VNLL.
A number of studies have therefore defined this structure in the very dorsal VNLL as a distinct nucleus, named the intermediate nucleus of the lateral lemniscus (INLL). Nevertheless, the borders and the extent of this structure are not clearly defined and show large species specific differences (Zook and Casseday, 1982; Saint Marie et al., 1997; Kelly et al., 2009; Ito et al., 2011). Using a transgenic mouse model, which expresses channelrhodopsin2 under the VGAT promoter and thus only in inhibitory, GABAergic or glycinergic neurons (Zhao et al., 2011), we show that all our neurons in the dorsal VNLL are inhibitory and therefore presumably not part of the excitatory, binaural INLL structure. We also identified a population of small neurons that are located dorsal of our investigated neurons might fulfill this criteria.

\section{Functional Heterogeneity of VNLL Neurons for Sound Processing}

The present study shows distinct variations in intrinsic and synaptic properties between the different population in the ventral and dorsal VNLL. In vivo several response patterns to sounds including onset, regular, sustained and chopper cells have been recorded in several species, however, with no clear correlation between response type and location within the VNLL (Batra and Fitzpatrick, 1997; Nayagam et al., 2006; 

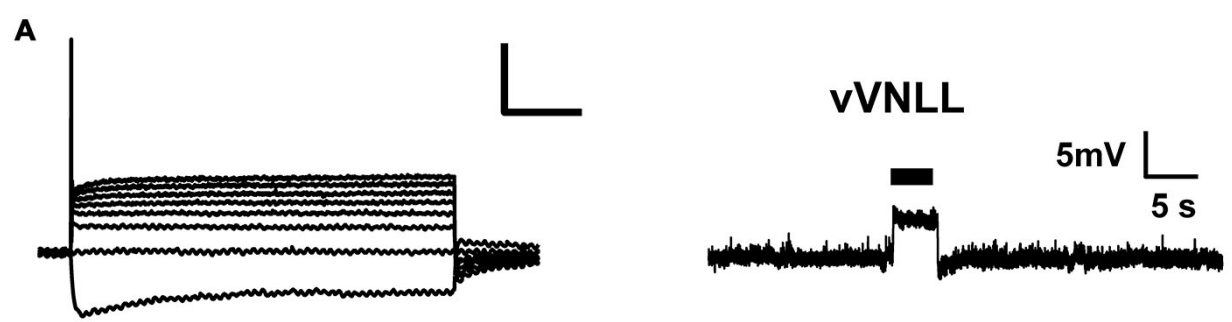

B

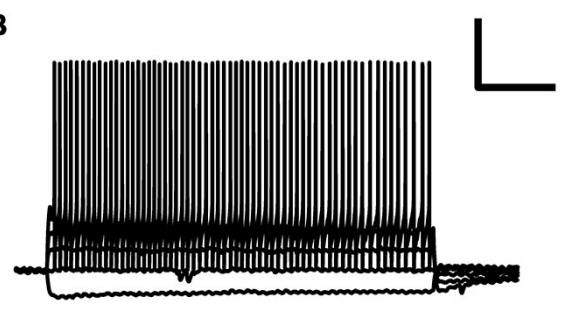

\section{dVNLL}
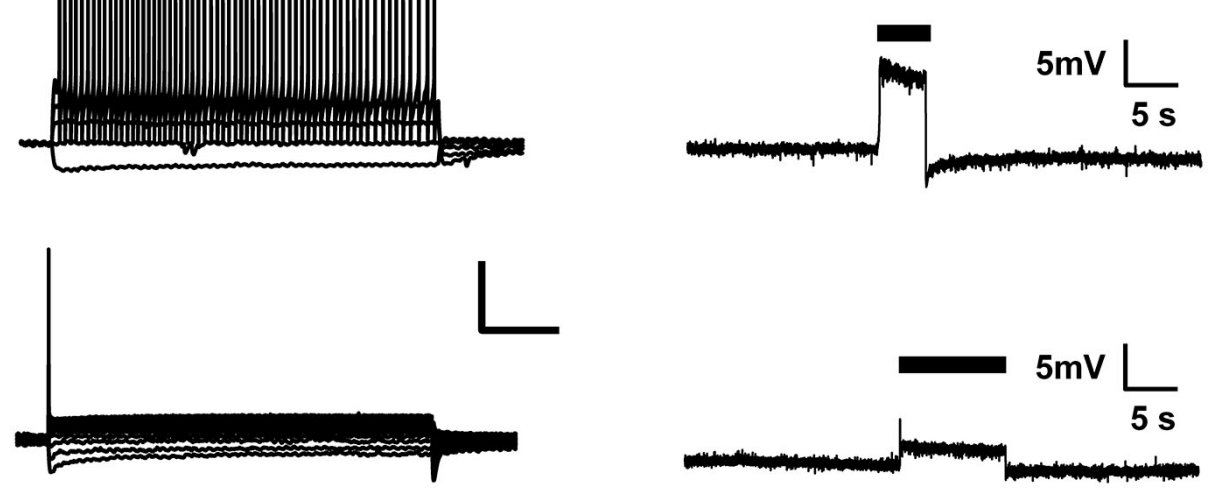

C

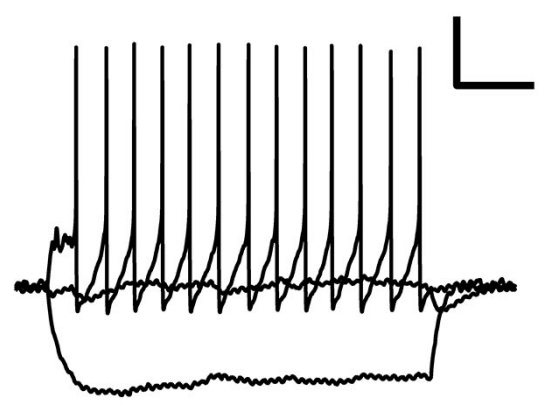

INLL

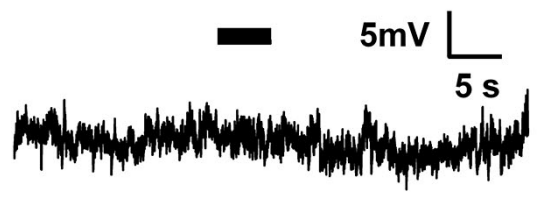

D

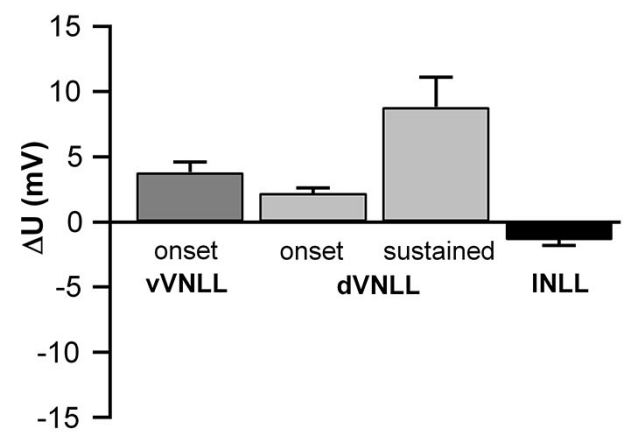

FIGURE 7 | Both vVNLL and dVNLL neurons are inhibitory. (A) Representative voltage trace of a vVNLL neuron to light stimulation (indicated by the black bar). The neuron depolarizes in response to the light pulse. The left panel depicts the corresponding firing pattern of the neuron. (B) Representative voltage traces of an onset-type and a sustained firing dVNLL neuron to light stimulation (indicated by the black bar). Both neurons depolarize in response to the light pulse. The left panels illustrate the corresponding firing patterns of the respective dVNLL neuron. (C) Representative voltage trace of an INLL neuron to light stimulation (indicated by the black bar). The left panel depicts a representative IV curve for INLL neurons. (D) Voltage deflection in response to light stimulation in VNLL and INLL neurons (VVNLL: $n=6$, dVNLL: sustained $n=6$, onset-type $n=9$; INLL: $n=8$ ). Data are obtained from seven mice. Scale bar insets: $200 \mathrm{~ms}, 20 \mathrm{mV}$. 
Zhang and Kelly, 2006). One possible explanation for this discrepancy is that the in vivo response patterns are mainly determined by the input properties whereas the intrinsic and synaptic properties are less influential. A similar lack of position with binaural response type of VNLL neurons was observed in most species examined. In the rat and cat most VNLL neurons are driven by contralateral stimulation with more pronounced ipsilateral inhibitory inputs in the rat but only weak influence by ipsilateral stimulation in the cat (Nayagam et al., 2006; Recio-Spinoso and Joris, 2014; Zhang and Kelly, 2006). A similar weak input from the ipsilateral ear is present in the mouse VNLL (unpublished observation). It is therefore unlikely that the two different response types in the dVNLL correspond to different groups of binaurality. We observed inhibitory, glycinergic inputs with similar characteristics to all neuron types and in all regions of the VNLL. Whether these inputs all derive from the previously characterized input of the ipsilateral MNTB (Kelly et al., 2009) or whether some of these inputs originate from other glycinergic nuclei, as e.g., the contralateral MNTB or the lateral nucleus of the trapezoid body, needs to be shown.

Finally, many anatomical and physiological observations suggest that the VNLL is involved in various forms of temporal processing of sound patterns (Covey and Casseday, 1991; Adams, 1997; Oertel, 1999; Nayagam et al., 2005). Especially the bushy cell-like neurons in the vVNLL that receive calyx-like inputs have received attention in this context. In the mouse we have identified a cluster of neurons in the ventrolateral part of the VNLL that receives a single calyx-like input that most likely originates from the octopus cells in the PVCN. Octopus cells preferably respond to broadband stimuli with precisely timed discharges (Rhode et al., 1983; Rouiller and Ryugo, 1984). Similarly, VNLLc neurons in bats respond with exceptionally sharply timed spikes to the onset of sounds (Covey and Casseday, 1991). Likewise, a population of VNLL neurons in other mammals shows precisely timed onset responses (Nayagam et al., 2005; Recio-Spinoso and Joris, 2014). These neurons are monaural and respond with one spike with short latencies precisely locked to the stimulus onset which makes these neurons suited to accurately encode temporal information (Recio-Spinoso and Joris, 2014). In accordance with this, ventrolateral VNLL neurons exhibit intrinsic properties that secure temporally precise information processing (Franzen et al., 2015). A recent model suggests that these neurons provide fast onset inhibition to neurons in other parts of the VNLL and the IC thereby suppressing

\section{REFERENCES}

Adams, J. C. (1997). Projections from octopus cells of the posteroventral cochlear nuceus to the ventral nucleus of the lateral lemniscus in cat and human. Audit. Neurosci. 3, 335-350.

Barnes-Davies, M., Barker, M. C., Osmani, F., and Forsythe, I. D. (2004). Kv1 currents mediate a gradient of principal neuron excitability across the tonotopic axis in the rat lateral superior olive. Eur. J. Neurosci. 19, 325-333. doi: 10.1111/j. 0953-816x.2003.03133.x

Batra, R., and Fitzpatrick, D. C. (1997). Neurons sensitive to interaural temporal disparities in the medial part of the ventral nucleus of the lateral lemniscus. J. Neurophysiol. 78, 511-515. spectral splatter (Spencer et al., 2015). Indeed, a previous study illustrates that a subpopulation of VNLL neurons receives a large preceding inhibition which strongly influences first spike latency (Nayagam et al., 2005; Spencer et al., 2015). The fast membrane and synaptic properties of the onset-type neurons of the dVNLL would be ideally suited to precisely integrate the fast preceding inhibition with several excitatory inputs. Nevertheless, in none of our recordings from dVNLL neurons we were able to detect an extremely large inhibitory input, as proposed in the model study (Spencer et al., 2015). It is possible that these large inhibitory inputs are received by a special type of neuron that is not preserved in the brain slice preparation. Alternatively, our stimulation method could be less effective in stimulating these inputs. Using the VGAT-channelrhodopsin mouse model could provide more information about this intrinsic inhibitory circuit.

\section{CONCLUSION}

We found distinct populations of neurons with different biophysical and synaptic properties in the dorsal and ventral parts of the VNLL. One neuron type in the ventrolateral VNLL displays onset-type response pattern and calyx-like excitatory input properties very similar to MNTB neurons. These properties makes these neurons likely candidates for providing temporally precise and fast feed-forward inhibition to target cells within the VNLL and to the IC. In contrast onset-type dVNLL neurons with large $I_{h}$ and short membrane time constants might contribute to the analysis of temporal sound pattern by integrating excitatory and inhibitory inputs on a fast time scale.

\section{AUTHOR CONTRIBUTIONS}

$\mathrm{VB}, \mathrm{FC}$ and UK conception and design of research; VB, FC and EG performed experiments; VB, FC and UK analyzed data; $\mathrm{VB}, \mathrm{FC}$ and UK interpreted results of experiments; VB, FC and EG prepared figures; VB, FC, EG and UK drafted and revised manuscript, and approved final version of manuscript.

\section{FUNDING}

This work was supported by Deutsche Forschungsgemeinschaft (SFB 665, SPP1608) and NeuroCure.

Batra, R., and Fitzpatrick, D. C. (1999). Discharge patterns of neurons in the ventral nucleus of the lateral lemniscus of the unanesthetized rabbit. $J$. Neurophysiol. 82, 1097-1113.

Baumann, V., Lehnert, S., Leibold, C., and Koch, U. (2013). Tonotopic organization of the hyperpolarization-activated current $\left(\mathrm{I}_{\mathrm{h}}\right)$ in the mammalian medial superior olive. Front. Neural Circuits 7:117. doi: 10.3389/fncir.2013. 00117

Benson, C. G., and Cant, N. B. (2008). The ventral nucleus of the lateral lemniscus of the gerbil (meriones unguiculatus): organization of connections with the cochlear nucleus and the inferior colliculus. J. Comp. Neurol. 510, 673-690. doi: 10.1002/cne. 21820 
Berger, C., Meyer, E. M. M., Ammer, J. J., and Felmy, F. (2014). Large somatic synapses on neurons in the ventral lateral lemniscus work in pairs. J. Neurosci. 34, 3237-3246. doi: 10.1523/JNEUROSCI.366413.2014

Borst, J. G. G., and Soria van Hoeve, J. (2012). The calyx of held synapse: from model synapse to auditory relay. Annu. Rev. Physiol. 74, 199-224. doi: 10. 1146/annurev-physiol-020911-153236

Brew, H. M., and Forsythe, I. D. (1995). Two voltage-dependent k+ conductances with complementary functions in postsynaptic integration at a central auditory synapse. J. Neurosci. 15, 8011-8022.

Covey, E., and Casseday, J. H. (1991). The monaural nuclei of the lateral lemniscus in an echolocating bat: parallel pathways for analyzing temporal features of sound. J. Neurosci. 11, 3456-3470.

Franzen, D. L., Gleiss, S. A., Berger, C., Kümpfbeck, F. S., Ammer, J. J., and Felmy, F. (2015). Development and modulation of intrinsic membrane properties control the temporal precision of auditory brain stem neurons. J. Neurophysiol. 113, 524-536. doi: 10.1152/jn.00601.2014

Glendenning, K. K., Brusno-Bechtold, J. K., Thompson, G. C., and Masterton, R. B. (1981). Ascending auditory afferents to the nuclei of the lateral leminscus. J. Comp. Neurol. 197, 673-703. doi: 10.1002/cne.901970409

Hassfurth, B., Magnusson, A. K., Grothe, B., and Koch, U. (2009). Sensory deprivation regulates the development of the hyperpolarization-activated current in auditory brainstem neurons. Eur. J. Neurosci. 30, 1227-1238. doi: 10. 1111/j.1460-9568.2009.06925.x

Huffman, R. F., and Covey, E. (1995). Origin of ascending projections to the nuclei of the lateral lemniscus in the big brown bat, Eptesicus fuscus. J. Comp. 357, $532-545$.

Indig, F. E., Diaz-Gonzalez, F., and Ginsberg, M. H. (1997). Analysis of the tetraspanin CD9-integrin alphaIIbbeta3 (GPIIb-IIIa) complex in platelet membranes and transfected cells. Biochem. J. 327, 291-298. doi: 10. 1042/bj3270291

Irfan, N., Zhang, H., and Wu, S. H. (2005). Synaptic transmission mediated by ionotropic glutamate, glycine and GABA receptors in the rat's ventral nucleus of the lateral lemniscus. Hear. Res. 203, 159-171. doi: 10.1016/j.heares.2004. 11.021

Ito, T., Bishop, D. C., and Oliver, D. L. (2011). Expression of glutamate and inhibitory amino acid vesicular transporters in the rodent auditory brainstem. J. Comp. Neurol. 519, 316-340. doi: 10.1002/cne.22521

Kelly, J. B., van Adel, B. A., and Ito, M. (2009). Anatomical projections of the nuclei of the lateral lemniscus in the albino rat (rattus norvegicus). J. Comp. Neurol. 512, 573-593. doi: 10.1002/cne.21929

Khurana, S., Liu, Z., Lewis, A. S., Rosa, K., Chetkovich, D., and Golding, N. L. (2012). An essential role for modulation of hyperpolarization-activated current in the development of binaural temporal precision. J. Neurosci. 32, 2814-2823. doi: 10.1523/JNEUROSCI.3882-11.2012

Kim, G., and Kandler, K. (2003). Elimination and strengthening of glycinergic/GABAergic connections during tonotopic map formation. Nat. Neurosci. 6, 282-290. doi: 10.1038/nn1015

Mathews, P. J., Jercog, P. E., Rinzel, J., Scott, L. L., and Golding, N. L. (2010). Control of submillisecond synaptic timing in binaural coincidence detectors by Kv1 channels. Nat. Neurosci. 13, 601-609. doi: 10.1038/nn.2530

Nayagam, D. A. X., Clarey, J. C., and Paolini, A. G. (2005). Powerful, onset inhibition in the ventral nucleus of the lateral lemniscus. J. Neurophysiol. 94, 1651-1654. doi: 10.1152/jn.00167.2005

Nayagam, D. A. X., Clarey, J. C., and Paolini, A. G. (2006). Intracellular responses and morphology of rat ventral complex of the lateral lemniscus neurons in vivo. J. Comp. Neurol. 498, 295-315. doi: 10.1002/cne.21058

Oertel, D. (1999). The role of timing in the brain stem auditory nuclei of vertebrates. Annu. Rev. Physiol. 61, 497-519. doi: 10.1146/annurev.physiol.61. 1.497

Recio-Spinoso, A., and Joris, P. X. (2014). Temporal properties of responses to sound in the ventral nucleus of the lateral lemniscus. J. Neurophysiol. 111, 817-835. doi: 10.1152/jn.00971.2011

Rhode, W. S., Oertel, D., and Smith, P. H. (1983). Physiological response properties of cells labeled intracellularly with horseradish peroxidase in cat ventral cochlear nucleus. J. Comp. Neurol. 213, 448-463. doi: 10.1002/cne.902130408
Riquelme, R., Saldaña, E., Osen, K. K., Ottersen, O. P., and Merchán, M. A. (2001). Colocalization of GABA and glycine in the ventral nucleus of the lateral lemniscus in rat: an in situ hybridization and semiquantitative immunocytochemical study. J. Comp. Neurol. 432, 409-424. doi: 10.1002/cne. 1111

Rouiller, E. M., and Ryugo, D. K. (1984). Intracellular marking of physiologically characterized cells in the ventral cochlear nucleus of the cat. J. Comp. Neurol. 225, 167-186. doi: 10.1002/cne.902250203

Saint Marie, R. L., and Baker, R. A. (1990). Neurotransmitter-specific uptake and retrograde transport of $[3 \mathrm{H}]$ glycine from the inferior colliculus by ipsilateral projections of the superior olivary complex and nuclei of the lateral lemniscus. Brain Res. 524, 244-253. doi: 10.1016/0006-8993(90)90698-b

Saint Marie, R. L., Shneiderman, A., and Stanforth, D. A. (1997). Patterns of gamma-aminobutyric acid and glycine immunoreactivities reflect structural and functional differences of the cat lateral lemniscal nuclei. J. Comp. Neurol. 389, 264-276. doi: 10.1002/(sici)1096-9861(19971215)389:2<264::aid-cne6>3. 0. co;2-\#

Schofield, B. R., and Cant, N. B. (1997). Ventral nucleus of the lateral lemniscus in guinea pigs: cytoarchitecture and inputs from the cochlear nucleus. J. Comp. Neurol. 379, 363-385. doi: 10.1002/(sici)1096-9861(19970317)379:3<363::aidcne4>3.0.co;2-1

Spencer, M. J., Nayagam, D. A. X., Clarey, J. C., Paolini, A. G., Meffin, H., Burkitt, A. N., et al. (2015). Broadband onset inhibition can suppress spectral splatter in the auditory brainstem. PLoS One 10:e126500. doi: 10.1371/journal.pone. 0126500

Vater, M., and Feng, A. S. (1990). Functional organization of ascending and descending connections of the cochlear nucleus of horseshoe bats. J. Comp. Neurol. 292, 373-395. doi: 10.1002/cne.902920305

Wahl-Schott, C., and Biel, M. (2009). HCN channels: structure, cellular regulation and physiological function. Cell. Mol. Life Sci. 66, 470-494. doi: 10. 1007/s00018-008-8525-0

Walcher, J., Hassfurth, B., Grothe, B., and Koch, U. (2011). Comparative posthearing development of inhibitory inputs to the lateral superior olive in gerbils and mice. J. Neurophysiol. 106, 1443-1453. doi: 10.1152/jn.01087.2010

Willard, F. H., and Ryugo, D. K. (1983). "Anatomy of the central auditory system," in The Auditory Psychobiology of the Mouse, ed. J. F. Willott (Springfield, IL: Charles Thomas), 201-304.

Wu, S. H. (1999). Physiological properties of neurons in the ventral nucleus of the lateral lemniscus of the rat: intrinsic membrane properties and synaptic responses. J. Neurophysiol. 81, 2862-2874.

Yavuzoglu, A., Schofield, B. R., and Wenstrup, J. J. (2011). Circuitry underlying spectrotemporal integration in the auditory midbrain. J. Neurosci. 31, 14424-14435. doi: 10.1523/JNEUROSCI.3529-11.2011

Zhang, H., and Kelly, J. B. (2006). Responses of neurons in the rat's ventral nucleus of the lateral lemniscus to amplitude-modulated tones. J. Neurophysiol. 96, 2905-2914. doi: 10.1152/jn.00481.2006

Zhao, M., and Wu, S. H. (2001). Morphology and physiology of neurons in the ventral nucleus of the lateral lemniscus in rat brain slices. J. Comp. Neurol. 433 , 255-271. doi: 10.1002/cne.1139

Zhao, S., Ting, J. T., Atallah, H. E., Qiu, L., Tan, J., Gloss, B., et al. (2011). Cell-type specific optogenetic mice for dissecting neural circuitry function. Nat. Methods 8, 745-752. doi: 10.1038/nmeth.1668

Zook, J. M., and Casseday, J. H. (1982). Cytoarchitecture of auditory system in lower brainstem of the mustache bat, pteronotus parnellii. J. Comp. Neurol. 207, 1-13. doi: 10.1002/cne.902070102

Conflict of Interest Statement: The authors declare that the research was conducted in the absence of any commercial or financial relationships that could be construed as a potential conflict of interest.

Copyright (c) 2015 Caspari, Baumann, Garcia-Pino and Koch. This is an open-access article distributed under the terms of the Creative Commons Attribution License (CC $B Y)$. The use, distribution and reproduction in other forums is permitted, provided the original author(s) or licensor are credited and that the original publication in this journal is cited, in accordance with accepted academic practice. No use, distribution or reproduction is permitted which does not comply with these terms. 